\title{
Corneoscleral Coat
}

National Cancer Institute

\section{Source}

National Cancer Institute. Corneoscleral Coat. NCI Thesaurus. Code C32371.

The outermost layer of the eyeball consisting of both the sclera and the cornea. 